\title{
Feasibility of Trypsin Digestion as a Sample Preparation for Daptomycin Quantification in Murine Skeletal Muscles
}

\author{
Yusuke Sakai, ${ }^{a}$ Eriko Murakami, ${ }^{b}$ Hideo Kato, ${ }^{c}$ Kaname Ohyama,${ }^{d}$ Yukihiro Esaka,,${ }^{a, b}$ \\ Takuhei Yamamoto, ${ }^{b}$ Mao Hagihara, ${ }^{c}$ Hiroshige Mikamo, ${ }^{e}$ and Bunji Uno*,a,b \\ ${ }^{a}$ The United Graduate School of Drug Discovery and Medical Information Sciences, Gifu University; 1-1 Yanagido, \\ Gifu 501-1193, Japan: ${ }^{b}$ Gifu Pharmaceutical University; 1-25-4 Daigaku-nishi, Gifu 501-1194, Japan: ${ }^{c}$ Department \\ of Pharmacy, Aichi Medical University School of Hospital; 1-1 Yazakokarimata, Nagakute, Aichi 480-1195, Japan: \\ ${ }^{d}$ Department of Pharmacy Practice, Graduate School of Biomedical Sciences, Nagasaki University; 1-7-1 Sakamoto- \\ machi, Nagasaki 852-8501, Japan: and ${ }^{e}$ Department of Infection Control and Prevention, Aichi Medical University \\ School of Hospital; 1-1 Yazakokarimata, Nagakute, Aichi 480-1195, Japan.
}

Received November 30, 2018; accepted January 10, 2019

\begin{abstract}
It is important to evaluate the amount of daptomycin (DAP) distributed to skeletal muscles to elucidate the mechanisms related to penetration and side effects, such as myopathies. However, no attempt has been made to measure DAP concentrations in skeletal muscles. The study's aim to investigate the feasibility of trypsin digestion, as a muscle sample preparation technique for the determination of DAP in murine skeletal muscle, was evaluated in conjunction with a conventional HPLC-UV analysis. Compared with trypsin digestion, DAP was less recovered from spiked skeletal muscle by the conventional extraction, including homogenization, centrifugation, and filtration, because of its incorporation into the muscle protein. On the other hand, a sample preparation technique involving enzymatic digestion employing trypsin fully recovered DAP from the spiked skeletal muscle. Based on the spike recovery assay results, we proposed an efficient muscle sample preparation method involving trypsin digestion. HPLC analysis in conjunction with the sample preparation method has successfully determined DAP concentrations of skeletal muscles collected from mice administrated subcutaneously with DAP. The proposed method is suitable for application to investigations that include animal experiments on drug migration into muscle and mechanism underlying skeletal muscle injury as a side reaction, such as myopathies, of DAP therapy.
\end{abstract}

Key words daptomycin; murine skeletal muscle; trypsin digestion

\section{INTRODUCTION}

Daptomycin (DAP) is a Streptomyces roseosporus-derived lipopeptide antibiotic that exhibits rapid and concentrationdependent bactericidal activity against Gram-positive bacteria including methicillin-resistant Staphylococcus aureus. ${ }^{1-3)}$ A few studies have identified unique bactericidal mechanisms of DAP, as well as so-called calcium-dependent antibiotic, such as permeabilization and depolarization of the bacterial cell membrane, ${ }^{4,5}$ being different from structurally similar lipopeptides such as tsushimycin as well as amphomycin and laspartomycin that inhibit peptidoglycan biosynthesis. ${ }^{4,5}$ However, the activity mechanisms involved in penetration of DAP in tissues, such as skin and muscle, are not fully understood. On the other hand, it is well recognized that the characteristic toxicity of DAP therapy is caused by myopathies, ${ }^{6-9)}$ specifically appears on the skeletal muscle, ${ }^{8)}$ and is induced by an overdose $^{8)}$ or short interval administration of DAP.9) As can be seen in a few case reports, there is also a concern about rhabdomyolysis, which is rare but serious, as a side effect of DAP therapy. ${ }^{10-13)}$ However, few factors that cause side effects have been identified. Kostrominova et al. suggested that the early effects of DAP on skeletal muscles of rats are due to the loss of sarcolemmal integrity based on the results of the histopathological examination which used immunostaining technique for the muscle injury of rats that were administered with DAP. ${ }^{14)}$ Once-daily dosing of DAP has also proved valid to minimize skeletal muscle effects. ${ }^{9)}$ However, it has been shown that the concentration of DAP in plasma is not associated with both increases in serum creatine phosphokinase activity and the incidence of myopathy. ${ }^{9}{ }^{9}$ Therefore, a method for accurate quantitation of DAP in skeletal muscle is essential for basic research using animals to study drug penetration and distribution properties to the muscle and the mechanism of the skeletal muscle injury as a side effect of DAP therapy.

Analytical studies on the quantification of DAP have been conducted to develop a method for the determination of DAP in plasma and serum that uses HPLC and ultra-HPLC with UV detection, ${ }^{15-18)}$ LC-MS, ${ }^{19)}$ and LC-tandem MS. ${ }^{20,21)}$ These methods rely on quite similar approaches to sample preparation for plasma and serum matrixes, such as solid-phase extraction and deproteinization with methanol or acetonitrile followed by centrifugation. ${ }^{15-21)}$ However, data on the tissue distribution of DAP are sparse and are confined to a pharmacokinetics study using rat skin tissue ${ }^{22)}$ and a clinical study using crushed human bone. ${ }^{23)}$ The centrifuged supernatants obtained from homogenization of the tissues followed by lengthy and repeated extraction using aqueous buffer solutions were then used for HPLC measurements of DAP in the tissues. ${ }^{22,23)}$ However, few details of the extractability of the drug from the homogenized skin-tissue suspension and crushed human bone before HPLC analysis are available.

We tried to determine the amount of DAP in muscle samples after administration in a preliminary experiment conducted on the use of a DAP loading dose in a murine infection model. ${ }^{24)}$ However, the peak corresponding to DAP was al- 
most undetectable on the chromatogram of the muscle sample obtained by the conventional extraction with homogenization and sonication, which is widely accepted as a sample preparation method for the analysis of a drug in biological solid matrixes. Therefore, a more effective pretreatment method for a skeletal muscle sample might enable us to quantify DAP distributed in the muscle by HPLC easily and accurately. As far as we know, no attempt has been made to measure DAP concentrations in skeletal muscles.

In this study, the feasibility of trypsin digestion as an efficient sample preparation technique for the bioanalysis of DAP in murine skeletal muscles was evaluated to gain more insight into drug migration into muscles and the mechanism underlying the side effect of skeletal muscle injury. The proposed method was applied to the determination of DAP in femoral muscle samples collected from mice which were administered with single subcutaneous dose of DAP.

\section{MATERIALS AND METHODS}

Chemicals DAP was purchased from Funakoshi Co., Ltd. (Tokyo, Japan) and was used without further purification. The DAP was subdivided into $200 \mu \mathrm{L}$ sample tubes as a $100 \mu \mathrm{M}$ aqueous solution and was stored in a freezer to achieve the desired concentration immediately before each experiment. Vancomycin hydrochloride from Streptomyces orientalis received from Sigma-Aldrich Co. (St. Louis, MO, U.S.A.) was used. The $100 \mu \mathrm{M}$ stock solution of vancomycin was prepared and stored in a freezer. Analytical grade 4-nitrophenol was commercially available from Kishida Chemical Co., Ltd. (Osaka, Japan) and was used without further purification as an internal standard for HPLC analysis. HPLC-grade acetonitrile used for HPLC eluent was purchased from Kanto Chemical Co., Inc. (Tokyo, Japan) and used as received without further purification. Ultrapure water produced by using a Milli-Q Academic A10 apparatus (Merck Millipore, Burlington, MA, U.S.A.) was used in all experiments.

Collections of Murine Skeletal Muscle Pathogen-free female ICR Swiss mice weighing approximately $22 \mathrm{~g}$ were purchased from Charles River Laboratories Japan, Inc. (Yokohama, Japan) and used throughout these experiments. The animals were maintained and used as per the National Research
Council recommendations and were provided food and water ad libitum. The study was reviewed and approved by the Aichi Medical University Hospital Institutional Animal Care and Use Committee.

After 10 mice were euthanized by $\mathrm{CO}_{2}$ asphyxiation without administering drugs, the thighs were immediately excised, followed by homogenization with $2 \mathrm{~mL}$ of sterile iced saline $(0.9 \%(\mathrm{w} / \mathrm{v}))$, and was used as analyte-free femoral muscle for drug recovery experiments. The homogenized analyte-free muscle was cut into pieces weighing approximately $0.2 \mathrm{~g}$ (wet weight); one piece was added to a pre-weighed $2 \mathrm{~mL}$ tube (Watson), and the exact weight was determined. The tube was stored at $-80^{\circ} \mathrm{C}$ until use. Another eight mice were injected subcutaneously with $100 \mathrm{mg} / \mathrm{kg}$ DAP in $200 \mu \mathrm{L}$ of saline, and five of them and the other three were sacrificed at 2 and $1 \mathrm{~h}$, respectively, after drug administration to collect the femoral muscles. The muscles were harvested in the same manner as the analyte-free muscles and were frozen at $-80^{\circ} \mathrm{C}$ until analysis.

Conventional Extraction Procedure Conventional liquid-solid extraction coupled with homogenization or sonication is still widely accepted as the most practical sample preparation method for the analysis of organic compounds, such as drugs, in biological solid matrixes. ${ }^{22,23)}$ It is well recognized that the use of a mixed solution consisting of chloroform and water efficiently extract water-soluble drugs, such as DAP and vancomycin hydrochloride, into the supernatant's aqueous layer separated from fat-soluble components, which is known as the Bligh-Dyer extraction method. ${ }^{25}$ ) We used this method as the conventional extraction procedure, as illustrated in Fig. 1 (P1) for the thorough and detailed experimental procedures. Methanol $(600 \mu \mathrm{L})$ and chloroform $(300 \mu \mathrm{L})$ were added to the tube $(2 \mathrm{~mL})$ containing exactly weighted muscle homogenate, which was then sealed with a cap and mixed on a vortex mixer for $10 \mathrm{~min}$. Subsequently, the contents of the tube were vortex mixed with $300 \mu \mathrm{L}$ chloroform and $400 \mu \mathrm{L}$ water for $30 \mathrm{~s}$ and then centrifuged at $3000 \times \boldsymbol{g}$ for $5 \mathrm{~min}$ to extract the drugs into the aqueous phase. The supernatant water $(1.0 \mathrm{~mL})$ was aspirated by using a pipette, followed by evaporation to dryness by using an evaporator. The residue dissolved in $50 \mu \mathrm{L}$ of water containing an internal standard was applied to an Ultrafree-MC $(0.22 \mu \mathrm{m}$ pore size $)$ filter

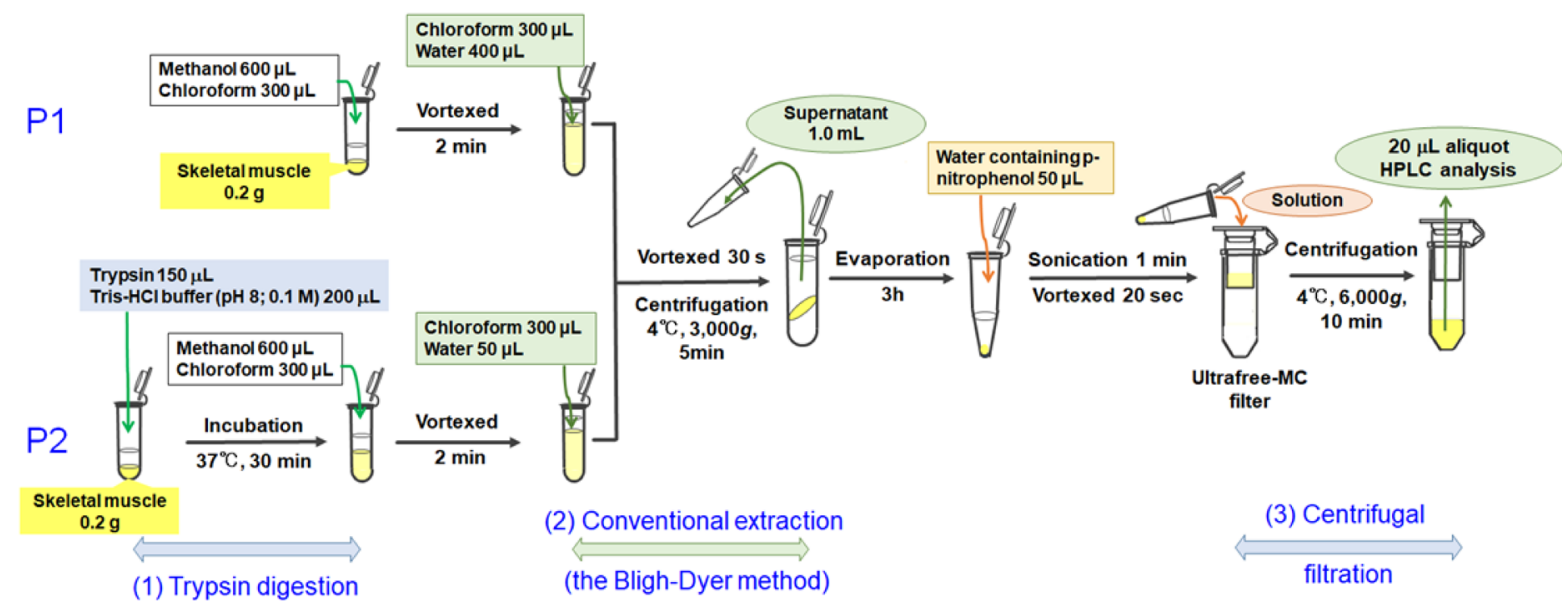

Fig. 1. Experimental Protocol for HPLC Analysis of DAP in Skeletal Muscle Samples

Sample preparation procedures of the conventional extraction (P1) and those involving trypsin digestion (P2). (Color figure can be accessed in the online version.) 
available from Merck Millipore by using a centrifuge set at $6000 \times \boldsymbol{g}$ at $4^{\circ} \mathrm{C}$ for $10 \mathrm{~min}$. A $20 \mu \mathrm{L}$ aliquot of the filtrate was injected onto the HPLC column.

Extraction Procedure Coupled with Trypsin Digestion Trypsin-ethylenediaminetetraacetic acid (EDTA) solution without phenol red, containing $0.5 \mathrm{w} / \mathrm{v} \%$ trypsin and $5.3 \mathrm{mM}$ EDTA.2Na, was purchased from FUJIFILM Wako Pure Chemical Corporation (Osaka, Japan) and used as a trypsin standard solution without further treatment. The optimal conditions for trypsin digestion of the murine skeletal muscles are shown in Fig. 1 (P2). The trypsin standard solution $(150 \mu \mathrm{L})$ and $0.1 \mathrm{M}$ Tris- $\mathrm{HCl}$ buffer $(\mathrm{pH} 8)$ solution $(200 \mu \mathrm{L})$ were added to the tube $(2 \mathrm{~mL})$ containing exactly weighed muscle homogenate, which was then sealed with a cap and incubated at $37^{\circ} \mathrm{C}$ for $30 \mathrm{~min}$. Subsequently, the contents of the tube were vortex mixed to yield a homogeneous suspension, followed by the conventional extraction, as illustrated in Fig. 1.

HPLC Analyses A Model 10AD VP HPLC system (Shimadzu, Kyoto, Japan) equipped with a DGU-12A degasser, a CLASS M10A (SPD-MXA) photodiode array detector, and a Model 7125 syringe-loading sample injector (Rheodyne, Cotati, CA, U.S.A.) was used. The HPLC separations were performed on an Inertsil ${ }^{\circledR}$ ODS-3 $(150 \times 4.6 \mathrm{~mm}$ i.d., $5 \mu \mathrm{m}$ particle size) column (GL Sciences Inc., Tokyo, Japan) with mobile phases of $0.01 \mathrm{M}$ phosphate buffer $(\mathrm{pH} 2.1)$ containing $36 \%$ acetonitrile and of $0.01 \mathrm{M}$ phosphate buffer ( $\mathrm{pH}$ 7.2) containing $10 \%$ acetonitrile for DAP and vancomycin, respectively, at a flow rate of $1.0 \mathrm{~mL} / \mathrm{min}$ at $40^{\circ} \mathrm{C}$. The column effluent was monitored at 221 and $280 \mathrm{~nm}$ for DPA and vancomycin, respectively, and the peak analyses were performed by using a Class M10A system (Shimadzu).

Calibration Curve A calibration curve was created by plotting the peak height ratios relative to an internal standard against the various concentrations of the standard DAP solutions. The limit of detection, defined as the lowest concentration that gave a signal-to-noise ratio of at least 3 , was found to be $0.3 \mu \mathrm{M}$ for DAP. The limit of quantification defined as the drug sample concentration meeting prespecified requirements for precision within $20 \%$ was found to be $0.75 \mu \mathrm{M}$. The calibration curve was linear across the examined dynamic range from 0.75 to $10 \mu \mathrm{M}(n=6, r=0.992)$. The relative standard deviation (RSD) values of the UV responses of DAP in 6 trials were $12.86,6.76$, and $2.18 \%$ for standard samples containing $0.75,2.5$, and $7.5 \mu \mathrm{M}$ DAP, respectively.

\section{RESULTS AND DISCUSSION}

Recovery Using the Conventional Extraction Method from Spiked Murine Skeletal Muscles Water-soluble DAP and vancomycin hydrochloride were expected to be extracted into the aqueous layer of an extract solution from femoral muscle homogenates. We conducted the additional recovery assay of the muscle homogenates spiked with DAP and vancomycin as preliminary experiments. Figure 2 shows the dependence of recovery of the drugs upon time after spiking the muscles. Recovery was evaluated by measuring three replicates of the samples. The mean recoveries for vancomycin were $96-104 \%$ at all the times observed, whereas those for DAP exponentially reduced with the passage of time and converged to approximately $25 \%$. This implied that DAP can be incorporated into skeletal muscle in the manner characterized

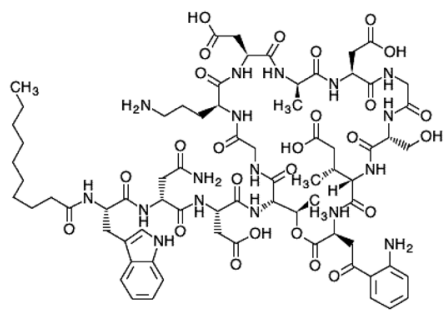

(a) daptomycin (DAP)

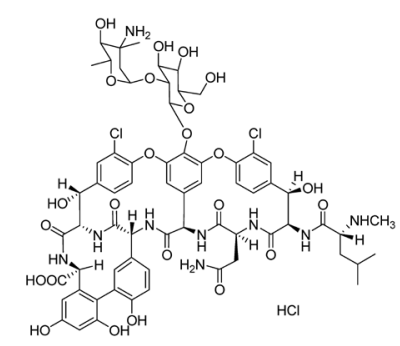

(b) vancomycin

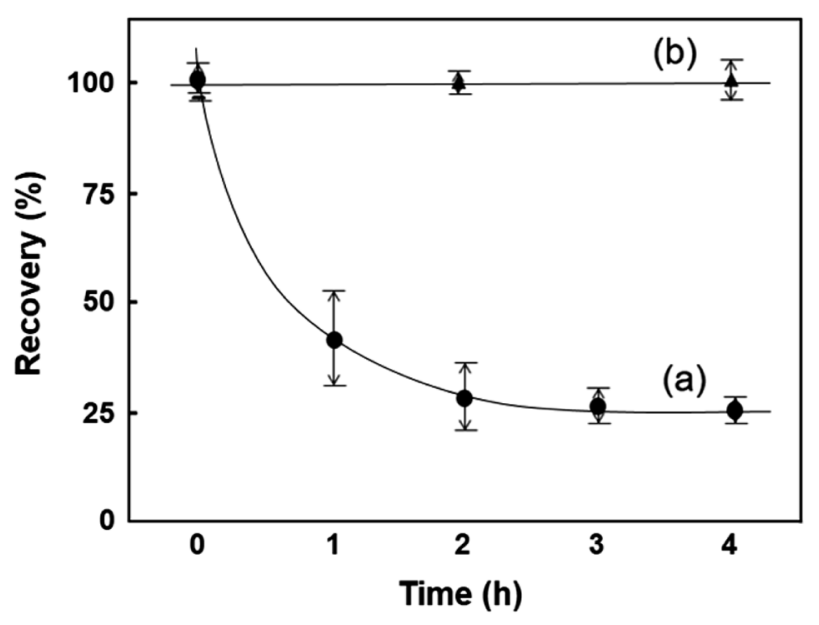

Fig. 2. Dependence of Recovery Ratios of DAP (a) and Vancomycin (b) on the Time to Start Conventional Extract after Spiking Murine Skeletal Muscles

The muscle samples were prepared by spiking analyte-free femoral muscle $(0.2 \mathrm{~g})$ with $20 \mu \mathrm{L}$ of the $100 \mu \mathrm{M}$ standard solution of the drug, and were treated according to procedure P1 shown in Fig. 1 . In the case of vancomycin, $0.1 \mathrm{M} \mathrm{HCl}$ $(100 \mu \mathrm{L})$ and $300 \mu \mathrm{L}$ water were added instead of $400 \mu \mathrm{L}$ water in the P1 procedure to dissociate vancomycin. Recovery calculated from the comparison with the peak height for standard solution prepared in the same concentration was evaluated by measuring three replicates of the samples. The error bars indicate the $95 \%$ confidence interval.

by somewhat specific binding unrecoverable by solvent extraction ascribed to partition equilibrium. Although approximately $92 \%$ DAP binds to plasma protein, ${ }^{7)}$ DAP is easily recovered from plasma by using the conventional pretreatments comprising deproteinization by methanol or acetonitrile, centrifugation, and extraction without the interference of the protein binding. ${ }^{15-21)}$ The physicochemistry underlying the strong incorporation of DAP into the muscle remains currently unknown; however, it is really interesting from the perspective of clinical analytical chemistry.

Enzymatic Skeletal Muscle Digestion with Trypsin To recover DAP incorporated into the skeletal muscles, we considered enzymatic digestion as an alternate tissue preparation technique, taking a hint from the efficient extraction of some lipophilic drugs from fat tissue digested with lipase. $\left.{ }^{26}\right)$ As trypsin specifically cleaves peptide chains at the carboxyl side of lysine or arginine, except when either is followed by proline, ${ }^{27)}$ it is conceivable that trypsin does not inactivate lipopeptide DAP. It has been demonstrated that DAP is not susceptible to hydrolysis by trypsin despite that diverse proteases such as chymotrypsin and type I proteases hydrolyze it. ${ }^{28)}$ Additionally, it is well recognized that trypsin is inhibited by calcium and magnesium ions. Therefore, we employed a trypsin solution containing EDTA as the most suitable enzyme for digestion of skeletal muscles. 
Trypsin digestion conditions were optimized by using the homogenized samples of skeletal muscle left for $3 \mathrm{~h}$ at a refrigerator after infusion of a DAP solution. Figure 3a shows recoveries of DAP from the muscle samples plotted against amounts of the trypsin standard solution used for the muscle digestion at $37^{\circ} \mathrm{C}$ for $10 \mathrm{~min}$. The use of a trypsin standard solution of $>100 \mu \mathrm{L}$ produces an exponential increase in the recovery of DAP. The reason for this phenomenon is still unknown, but it suggests that DAP is instantaneously released from the collapsed structure of some proteins through cleavage of specific peptide bonds. On the other hand, the dependence of recovery of DAP from the skeletal muscle upon digestion time is illustrated in Fig. 3b. Complete dissolution of $0.2 \mathrm{~g}$ of murine muscle was obtained by digestion with a $150 \mu \mathrm{L}$ trypsin standard solution at $37^{\circ} \mathrm{C}$ for $30 \mathrm{~min}$. We note that DAP responses start to decrease after $1 \mathrm{~h}$ of digestion, as shown in Fig. 3b. As a result, digestion of $0.2 \mathrm{~g}$ of murine skeletal muscle at $37^{\circ} \mathrm{C}$ for a period of $30 \mathrm{~min}$ was determined to be the optimal digestion conditions for a $150 \mu \mathrm{L}$ trypsin standard solution, as shown in Fig. 1 (P2). In subsequent investigations, these conditions were employed.

Recoveries from the Skeletal Muscles Spiked with DAP We found that trypsin treatment is very effective for the extraction of DAP incorporated strongly into skeletal muscles. We conducted experiments for recovery of DAP from femoral muscles spiked with DAP. Figure 4 shows the experimental procedures for pretreatments of the muscle samples. A $20 \mu \mathrm{L}$ aliquot of $50 \mu \mathrm{M}$ DAP solution was added to the homogenate of analyte-free femoral muscle $(0.2 \mathrm{~g})$, which was stored in a refrigerator for $3 \mathrm{~h}$. The four samples were used for the conventional extraction experiments (Fig. 4A) and those involving trypsin digestion (Fig. 4C), respectively. In addition to this, the residual muscle after the conventional extraction was treated with trypsin followed by the conventional extraction again (Fig. 4B). The mean recovery data are also shown in Fig. 4. It is interesting that the sum of the collected amounts of $\mathrm{A}$ and $\mathrm{B}$ is comparable to the recovery amount of $\mathrm{C}$, which implies that more than two-thirds of DAP present in skeletal muscle (a)

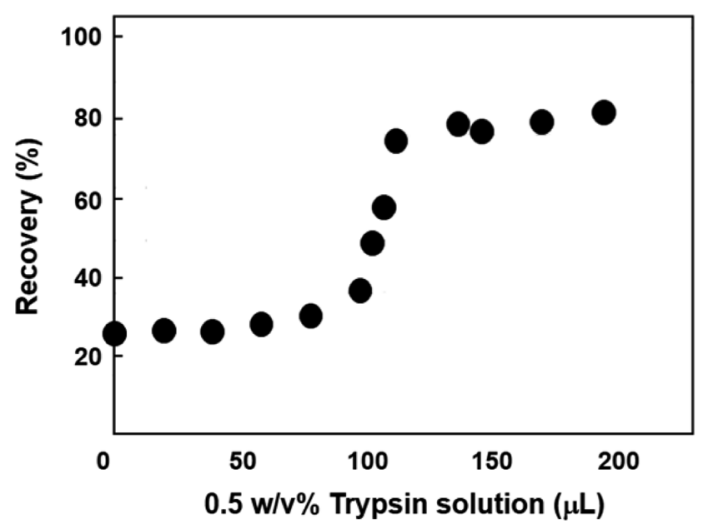

(b)

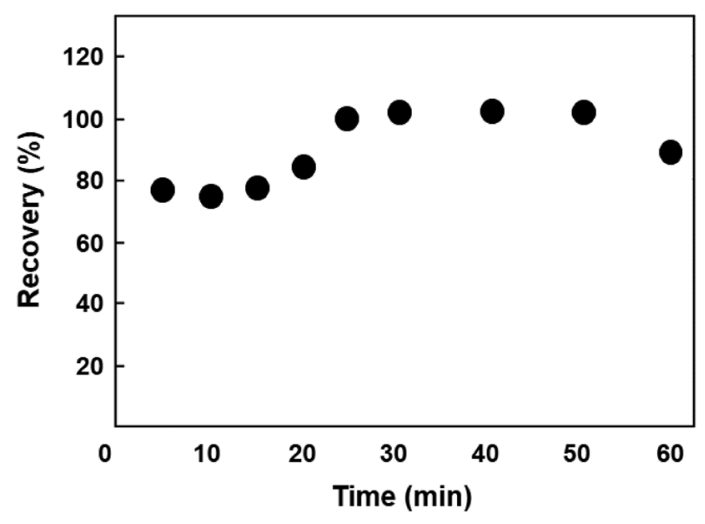

Fig. 3. Dependences of Recovery of DAP from the Muscle Spiked with DAP on the Amounts of Trypsin Standard Solution Used for Digestion (a) and on Incubation Time for Muscle Digestion (b)

The additional recovery samples were prepared by using analyte-free muscle $(0.2 \mathrm{~g})$ spiked with a $20 \mu \mathrm{L}$ aliquot of $50 \mu \mathrm{M}$ DAP standard solution, and treated with trypsin followed by the conventional extraction, as shown in Fig. 1. (a) Tryp$\sin$ was used for muscle digestion involving incubation for $10 \mathrm{~min}$ at $37^{\circ} \mathrm{C}$. (b) Incubation time was measured for muscle digestion with $150 \mu \mathrm{L}$ trypsin standard solution at $37^{\circ} \mathrm{C}$.

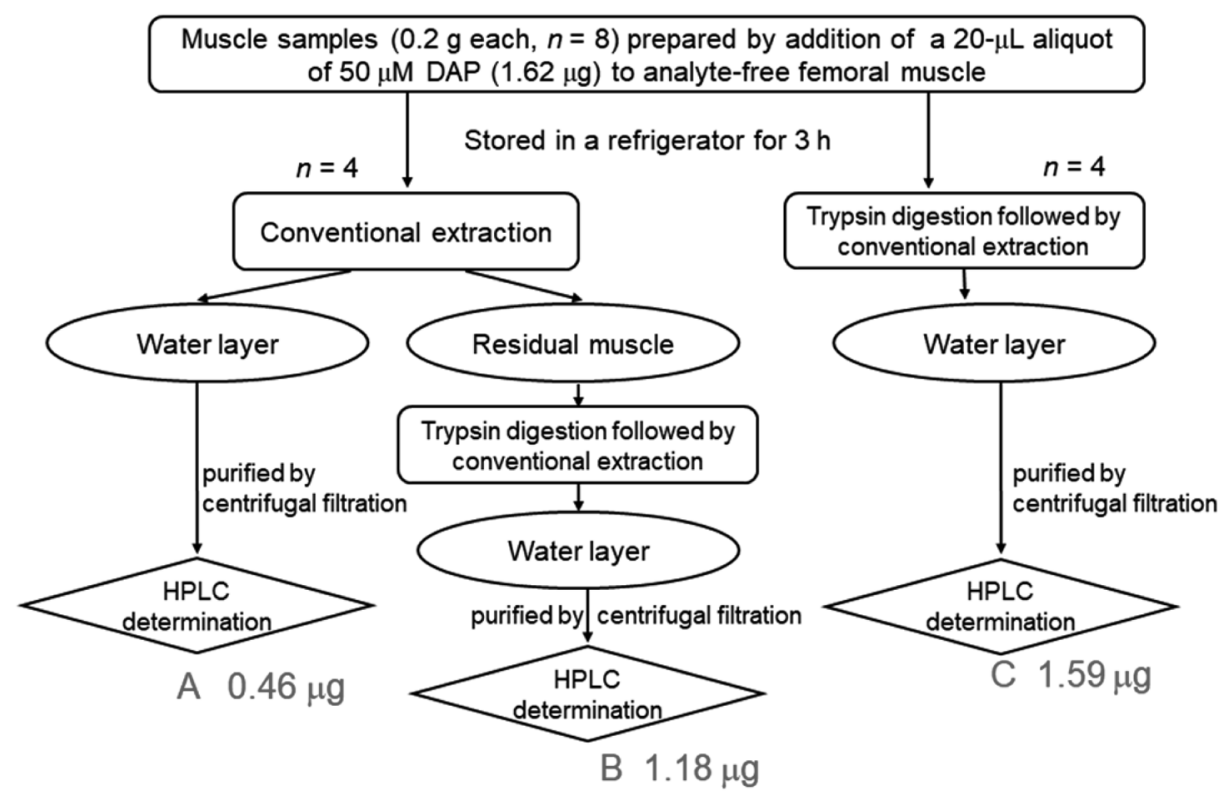

Fig. 4. Experimental Scheme for Recovery Assays from Skeletal Muscle Samples

The muscle samples were analyte-free femoral muscles spiked with $1.62 \mu \mathrm{g}$ DAP. The measured mean values $(n=4)$ are shown in gray. 
Table 1. Recovery Data and the RSD of DAP from Murine Skeletal Muscles Spiked with 0.064, 0.16, and 1.62 $\mu$ g DAP

\begin{tabular}{|c|c|c|c|c|c|c|c|}
\hline \multirow{2}{*}{ Addition amounts $(\mu \mathrm{g})$} & \multicolumn{5}{|c|}{ Recovery $(\%)^{a)}$} & \multirow{2}{*}{ S.D. } & \multirow{2}{*}{ RSD (\%) } \\
\hline & 1 & 2 & 3 & 4 & Mean & & \\
\hline $0.064^{b)}$ & 75.2 & 42.3 & 87.3 & 52.8 & 64.4 & 20.52 & 31.87 \\
\hline $0.16^{c)}$ & 86.3 & 84.4 & 103.1 & 94.3 & 92.0 & 8.54 & 9.28 \\
\hline $1.62^{d)}$ & 97.5 & 99.5 & 101.1 & 100.3 & 99.6 & 1.54 & 1.55 \\
\hline
\end{tabular}

a) Data obtained from HPLC analysis after sample pretreatment P2 shown in Fig. 1. b) Prepared by spiking a $20 \mu \mathrm{L}$ aliquot of a $2.0 \mu \mathrm{M}$ DAP standard solution. The concentration of this muscle sample is calculated as $0.32 \mu \mathrm{g} / \mathrm{g}$ (muscle). $c$ ) Prepared by spiking a $20 \mu \mathrm{L}$ aliquot of a $5.0 \mu \mathrm{M}$ DAP standard solution. The concentration of this muscle sample is calculated as $0.80 \mu \mathrm{g} / \mathrm{g}$ (muscle). d) Prepared by spiking a $20 \mu \mathrm{L}$ aliquot of a $50 \mu \mathrm{M}$ DAP standard solution. The concentration of this muscle sample is calculated as $8.0 \mu \mathrm{g} / \mathrm{g}$ (muscle). The mean value shown in Fig. $4 \mathrm{C}$ was obtained from these experiments.

Table 2. DAP Concentrations in Murine Femoral Muscles Collected $2 \mathrm{~h}$ after the Single Subcutaneous Administration of DAP (100 mg/kg)

\begin{tabular}{|c|c|c|c|c|c|c|c|c|}
\hline \multirow{2}{*}{ Procedure $^{a)}$} & \multicolumn{6}{|c|}{ Measured values of DAP $(\mu \mathrm{g} / \mathrm{g})^{b)}$} & \multirow{2}{*}{ S.D. } & \multirow{2}{*}{ RSD (\%) } \\
\hline & 1 & 2 & 3 & 4 & 5 & Mean & & \\
\hline A & 1.1 & 1.3 & 1.4 & 1.5 & 1.1 & 1.3 & 0.18 & 14.0 \\
\hline $\mathrm{B}$ & 1.5 & 1.9 & 2.2 & 1.8 & 1.4 & 1.8 & 0.32 & 18.2 \\
\hline $\mathrm{C}$ & 2.5 & 3.0 & 3.5 & 3.3 & 2.4 & 2.9 & 0.48 & 16.4 \\
\hline $\mathrm{C}(1 \mathrm{~h})^{c)}$ & 3.0 & 2.8 & 3.8 & - & - & 3.2 & 0.53 & 16.5 \\
\hline
\end{tabular}

a) Sample preparation procedures shown in Fig. 5. b) Measured values are indicated by the amount ( $\mu \mathrm{g})$ of DAP contained in the $1 \mathrm{~g}$ of muscle. $c$ ) Data obtained from femoral muscle collected $1 \mathrm{~h}$ after administration of DAP.

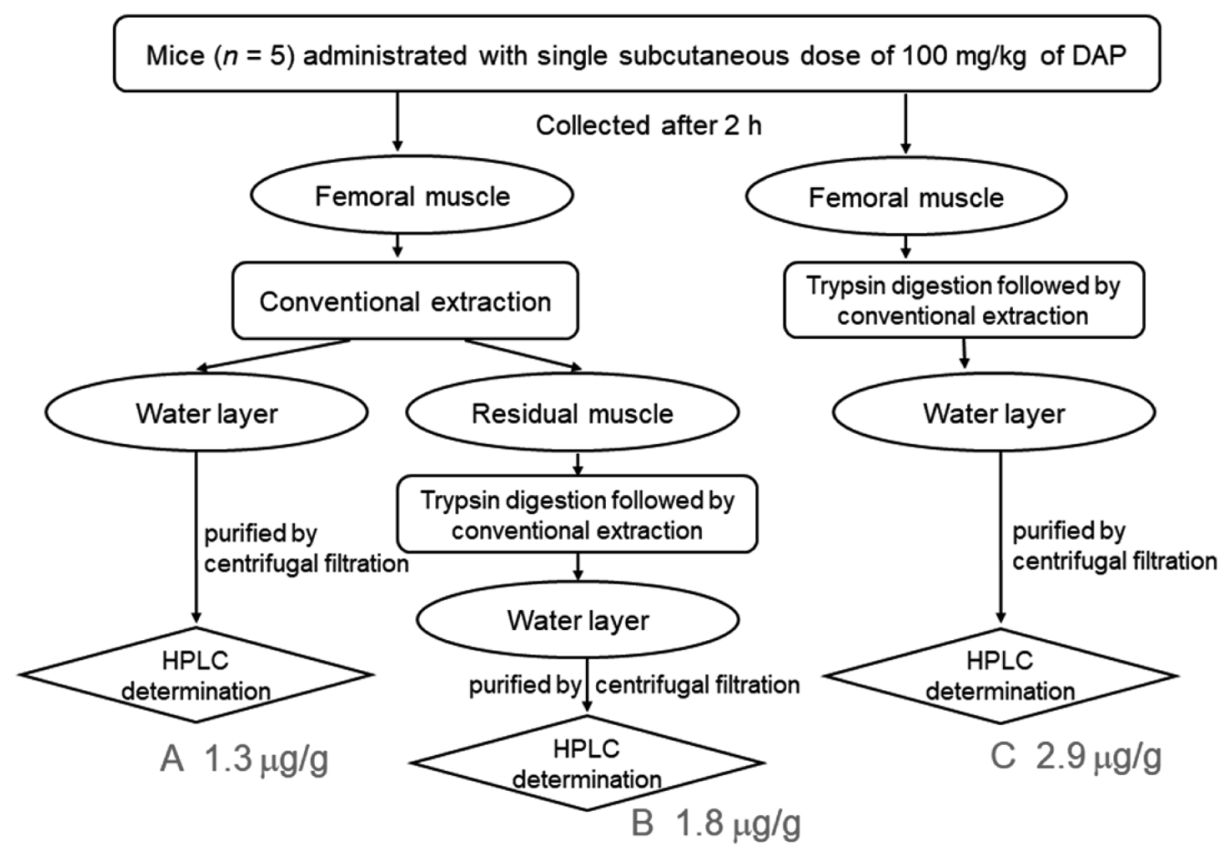

Fig. 5. Experimental Scheme for the HPLC Analyses of the Determination of DAP in Murine Skeletal Muscles Collected from Mice $(n=5)$ Administered a Single Subcutaneous Dose of $100 \mathrm{mg} / \mathrm{kg}$ DAP

strongly interacts with some muscle proteins and is difficult to extract without the digestive power of trypsin.

Table 1 shows the results of recovery assays for the repeated experiments performed using the homogenized femoral muscle samples infused at three different concentration levels in quadruplicate. The obtained recovery data, except for the case of the $0.064 \mu \mathrm{g}$ addition, satisfy the acceptance criteria for biochemical assays. The results show that the enzyme digestion employed in the extraction process contributed to the high and stable recovery of DAP $>0.8 \mu \mathrm{g} / \mathrm{g}$ (muscle) in murine skeletal muscle. Alternatively, a spike with $0.064 \mu \mathrm{g}$ DAP gave an unacceptable deviation, as presented in Table 1. The amount of addition converted into the final analyte concentration to be injected into the HPLC was $<0.8 \mu \mathrm{M}$, which is close to the quantification limit of the present HPLC-UV method $(0.75 \mu \mathrm{M})$. Therefore, pretreatment involving trypsin digestion appeared to contribute to precise quantification of DAP in murine skeletal muscle under the assumption that DAP transferred to the muscle through drug administration is in the same state as that spiked into the muscle directly.

DAP Determination in Skeletal Muscles of Mice Administered Subcutaneously Based on the conditions to perform complete digestion of the muscle and efficient recovery of DAP from the muscle, we proposed a pretreatment method for 
DAP determination in murine skeletal muscles, as is denoted P2 in Fig. 1. We conducted quantification experiments of DAP in the femoral muscles collected from mice administered with DAP according to the proposed method. Figure 5 shows the experimental scheme for pretreatments of the muscle samples collected $2 \mathrm{~h}$ after single subcutaneous administration of $100 \mathrm{mg} / \mathrm{kg}$ DAP, and the measured values are presented in Table 2.

Since all of the samples have individual differences and issues based on the localization of analytes in the muscle tissue, variability in the analysis results of in vivo muscle tissue samples is both expected and acceptable. The incurred DAP levels were found to be higher with the present method (C) than the values obtained by the muscle preparation using the conventional extraction (A). It was also found that the relationship in which the sum of $\mathrm{A}$ and $\mathrm{B}$ is equal to $\mathrm{C}$ is established for all the samples, respectively. This finding implies that trypsin treatment is essential to determine the precise concentration of DAP involving incorporation through interaction with some muscle proteins. On the other hand, the large changes in muscle concentration 1 to $2 \mathrm{~h}$ after administration may be expected as it is well recognized that DAP concentrations in plasma and serum show a clear decrease within a few hours. ${ }^{21,24)}$ However, both concentrations of DAP appeared to be comparable, as listed in Table 2 . These are only the results of a pilot experiment; further studies are needed using this proposed method to investigate the relationship between the drug concentration variances in plasma and muscle over time after administration. Although the dosage is more than amounts generally used in the administration experiments, the feasibility of DAP determination in the skeletal muscle is well illustrated.

\section{CONCLUSION}

The feasibility of enzymatic digestion as a sample preparation technique for bioanalysis of DAP in murine skeletal muscles was evaluated. Our results showed that trypsin digestion was an appropriate sample preparation method for analysis of DAP incorporated into skeletal muscle through interaction with some proteins. This sample preparation method involving trypsin digestion could be used to identify factors that might predispose some patients to develop DAP-related myopathies with the aid of a highly-sensitive analytical method for DAP. The strong incorporation into the muscle may be possibly associated with skeletal muscle injury of DAP therapy.

There are only a few attempts to employ enzymatic digestion in tissue sample preparation of drug molecules. ${ }^{26,29,30)} \mathrm{Yu}$ et al. have proposed that enzymatic digestion as an alternate tissue preparation technique for bioanalysis of drugs has extraction efficiency comparable to that of homogenization. ${ }^{29)}$ However, as far as we know, there have been no reports evaluating the feasibility of enzymatic digestion as a release technique from some muscle proteins interacting with the drug. This is the first report on the pretreatment of muscle sample using trypsin digestion. Further work to estimate the relationship between DAP concentrations in the serum and the skeletal muscle, and to identify the muscle proteins strongly interacting with DAP by using this muscle sample preparation technique is ongoing.
Acknowledgments The authors would like to thank Miss Chisato Hayakawa and Miss Hanae Katsukawa of Gifu Pharmaceutical University for their experimental assistances.

Conflict of Interest The authors declare no conflict of interest.

\section{REFERENCES}

1) Liu C, Mao Z, Yang M, Kang H, Liu H, Pan L, Hu J, Luo J, Zhou F. Efficacy and safety of daptomycin for skin and soft tissue infections: a systematic review with trial sequential analysis. Ther. Clin. Risk. Manag., 12, 1455-1466 (2016).

2) Schriever CA, Fernández C, Rodvold KA, Danziger LH. Daptomycin: a novel cyclic lipopeptide antimicrobial. Am. J. Health Syst. Pharm., 62, 1145-1158 (2005).

3) Mortin LI, Li T, Van Praagh AD, Zhang S, Alder JD. Rapid bactericidal activity of daptomycin against methicillin-resistant and methicillin-susceptible Staphylococcus aureus peritonitis in mice as measured with bioluminescent bacteria. Antimicrob. Agents Chemother., 51, 1787-1794 (2007).

4) Taylor SD, Palmer M. The action mechanism of daptomycin. Bioorg. Med. Chem., 24, 6253-6268 (2016).

5) Silverman JA, Perlmutter NG, Shapiro HM. Correlation of daptomycin bactericidal activity and membrane depolarization in Staphylococcus aureus. Antimicrob. Agents Chemother., 47, 2538-2544 (2003).

6) Livermore DM. Future directions with daptomycin. J. Antimicrob. Chemother., 62 (suppl. 3), iii41-iii49 (2008).

7) Sauermann R, Rothenburger M, Graninger W, Joukhadar C. Daptomycin: a review 4 years after first approval. Pharmacology, 81, 79-91 (2008).

8) Tally FP, Zeckel M, Wasilewski MM, Carini C, Berman CL, Drusano GL, Oleson FB Jr. Daptomycin: a novel agent for Gram-positive infections. Expert Opin. Investig. Drugs, 8, 1223-1238 (1999).

9) Oleson FB Jr, Berman CL, Kirkpatrick JB, Regan KS, Lai JJ, Tally FP. Once-daily dosing in dogs optimizes daptomycin safety. Antimicrob. Agents Chemother., 44, 2948-2953 (2000).

10) Sbrana F, Paolo AD, Pasanisi EM, Tagliaferri E, Arvia C, Puntoni M, Leonildi A, Bigazzi F, Danesi R, Rovai D, Tascini C, Menichetti F. Administration interval and daptomycin toxicity: a case report of rhabdomyolysis. J. Chemother., 22, 434-435 (2010).

11) Papadopoulos S, Ball AM, Liewer SE, Martin CA, Winstead PS, Murphy BS. Rhabdomyolysis during therapy with daptomycin. Clin. Infect. Dis., 42, e108-e110 (2006).

12) Kazory A, Dibadj K, Weiner ID. Rhabdomyolysis and acute renal failure in a patient treated with daptomycin. J. Antimicrob. Chemother., 57, 578-579 (2006)

13) King ST, Walker ED, Cannon CG, Finley RW. Daptomycin-induced rhabdomyolysis and acute liver injury. Scand. J. Infect. Dis., 46, 537-540 (2014)

14) Kostrominova TY, Hassett CA, Rader EP, Davis C, Larkin LM, Coleman S, Oleson FB, Faulkner JA. Characterization of skeletal muscle effects associated with daptomycin in rats. Muscle Nerve, 42, 385-393 (2010)

15) Martens-Lobenhoffer J, Kielstein JT, Oye C, Bode-Böger SM. Validated high performance liquid chromatography-UV detection method for the determination of daptomycin in human plasma. $J$. Chromatogr. B Analyt. Technol. Biomed. Life Sci., 875, 546-550 (2008).

16) Gikas E, Bazoti FN, Fanourgiakis P, Perivolioti E, Roussidis A, Skoutelis A, Tsarbopoulos A. Simultaneous quantification of daptomycin and rifampicin in plasma by ultra performance liquid chromatography: application to a pharmacokinetic study. J. Pharm. Biomed. Anal., 51, 901-906 (2010).

17) Baietto L, D’Avolio A, Pace S, Simiele M, Marra C, Ariaudo A, 
Perri GD, Rosa FGD. Development and validation of an UPLCPDA method to quantify daptomycin in human plasma and in dried plasma spots. J. Pharm. Biomed. Anal., 88, 66-70 (2014).

18) Naito T, Yamada T, Yagi T, Kawakami J. Simple and validated UHPLC method coupled to UV detection for determination of daptomycin in human plasma and urine. Biomed. Chromatogr., 28, 317-319 (2014)

19) Baietto L, D’Avolio A, Rosa FGD, Garazzino S, Michelazzo M, Ventimiglia G, Siccardi M, Simiele M, Sciandra M, Perri GD. Development and validation of a simultaneous extraction procedure for HPLC-MS quantification of daptomycin, amikacin, gentamicin, and rifampicin in human plasma. Anal. Bioanal. Chem., 396, 791-798 (2010).

20) Bazoti FN, Gikas E, Skoutelis A, Tsarbopoulos A. Development and validation of an ultra performance liquid chromatographytandem mass spectrometry method for the quantification of daptomycin in human plasma. J. Pharm. Biomed. Anal., 56, 78-85 (2011).

21) Verdier MC, Bentué-Ferrer D, Tribut O, Collet N, Revest M, Bellissant E. Determination of daptomycin in human plasma by liquid chromatography-tandem mass spectrometry. Clinical application. Clin. Chem. Lab. Med., 49, 69-75 (2011).

22) Matsumoto K, Kitaoka M, Kuroda Y, Ikawa K, Morikawa N, Sasaki J, Iketani O, Iwata S, Horino T, Hori S, Kizu J. Pharmacokinetics and skin-tissue penetration of daptomycin in rats. Clin. Pharmacol., 7, 79-82 (2015).

23) Montange D, Berthier F, Leclerc G, Serre A, Jeunet L, Berard M, Muret P, Vettoretti L, Leroy J, Hoen B, Chirouze C. Penetration of daptomycin into bone and synovial fluid in joint replacement. Antimicrob. Agents Chemother., 58, 3991-3996 (2014).

24) Kato H, Hagihara M, Murakami E, Suematsu H, Nishiyama N, Koizumi Y, Yamagishi Y, Uno B, Mikamo H. Considerations about the use of a loading dose of daptomycin in a neutropenic murine thigh infection model with methicillin-resistant Staphylococcus aureus infection. Chemotherapy, 63, 13-19 (2018).

25) Bligh EG, Dyer WJ. A rapid method of total lipid extraction and purification. Can. J. Biochem. Physiol., 37, 911-917 (1959).

26) Storey GC, Adams PC, Campbell RW, Holt DW. High-performance liquid chromatographic measurement of amiodarone and desethylamiodarone in small tissue samples after enzymatic digestion. $J$. Clin. Pathol., 36, 785-789 (1983).

27) Engelking L. Textbook of Veterinary Physiological Chemistry. Third ed., Academic Press, Boston, pp. 39-44 (2014).

28) D'Costa VM, Mukhtar TA, Patel T, Koteva K, Waglechner N, Hughes DW, Wright GD, Pascalea GD. Inactivation of the lipopeptide antibiotic daptomycin by hydrolytic mechanisms. Antimicrob. Agents Chemother., 56, 757-764 (2012).

29) Yu C, Penn LD, Hollembaek J, Li W, Cohen LH. Enzymatic tissue digestion as an alternative sample preparation approach for quantitative analysis using liquid chromatography-tandem mass spectrometry. Anal. Chem., 76, 1761-1767 (2004).

30) Posyniak A, Zmudzki J, Semeniuk S. Effects of the matrix and sample preparation on the determination of fluoroquinolone residues in animal tissues. J. Chromatogr. A, 914, 89-94 (2001). 